\title{
Pyogenic liver abscess caused by community-acquired multidrug resistance Pseudomonas aeruginosa
}

\author{
Authors \\ Mehmet Ulug ${ }^{1}$ \\ Ercan Gedik ${ }^{2}$ \\ Sadullah Girgin ${ }^{2}$ \\ Mustafa Kemal Celen ${ }^{3}$ \\ Celal Ayaz ${ }^{3}$ \\ ${ }^{1}$ BSK Anadolu Hospital, \\ Department of Infectious \\ Diseases and Clinic \\ Microbiology, 43100 \\ Kütahya, Turkey. \\ ${ }^{2}$ Dicle University Medical \\ School, Department of \\ General Surgery, 21280 \\ Diyarbakir, Turkey. \\ ${ }^{3}$ Dicle University Medical \\ School, Department of \\ Infectious Diseases and \\ Clinic Microbiology, 21280 \\ Diyarbakir, Turkey.
}

Submitted on: 03/04/2010 Approved on: 03/22/2010

\section{Correspondence to:} Mehmet Ulug, MD BSK Anadolu Hospital, Department of Infectious Diseases and Clinic Microbiology, 43100 Kütahya, Turkey Phone (mobile): +90-532-4475756 Fax: +90-274-2244433 E-mail: mehmetulug21@ yahoo.com

Pyogenic liver abscess (PLA) is a very uncommon liver disease affecting predominantly young males in the setting of intra-abdominal infection. ${ }^{1}$ PLA can be caused by several organisms. An uncommon case of PLA caused by multidrug resistant (MDR) Pseudomonas aeruginosa is described.

A previously healthy 28-year-old man was admitted to our clinic with a two weeks history of pain in the right upper abdomen, followed by fever, chills, and vomiting. On admission day, physical examination revealed that he was in pain and feverish $\left(38.6^{\circ} \mathrm{C}\right)$, and had hepatomegaly. Laboratory findings included total white blood cell (WBC) count of $14,800 / \mathrm{mm}^{3}$, erythrocyte sedimentation rate was $87 \mathrm{~mm} / \mathrm{h}$ with a C-reactive protein (CRP) measuring $63 \mathrm{mg} / \mathrm{dL}$ (range, $0-8 \mathrm{mg} / \mathrm{dL}$ ), and total bilirubin, alanine aminotransferase, aspartate aminotransferase, gamma glutamyl transpeptidase, alkaline phosphatase were within normal limits. Abdominal ultrasonography (US) showed solid mass at right hepatic lobe, which suggested a liver abscess, according to clinical findings. It was also confirmed by abdominal computed tomography (CT). The abscess was localized with transverse CT images and percutaneous drainage with 20 Fr gauge was performed. Fluid samples were sent for microbiological cultures. He was initially treated with ceftriaxone $2 \mathrm{~g}$ /day plus metronidazole $1 \mathrm{~g} /$ day with no improvement. After three days, the aspiration culture grew MDR $P$. aeruginosa. The bacteriological resistance results led us to change the initial treatment to imipenem $2 \mathrm{~g}$ /day plus amikacin $1.5 \mathrm{~g}$ /day for four weeks. The patient was discharged 21 days after admission without evidence of any complication.

Despite continuous improvement in image modalities, availability of potent antibiotics and advancement in the knowledge and treatment of PLA, morbidity and mortality remains high. Its incidence ranges from 0.008 to $0.0022 \%$ in hospitalized patients. ${ }^{2,3}$ The diagnosis of PLA was based on clinical findings and evidence from imaging studies, either abdominal US or CT. Treatment with intravenous antibiotics and application of catheter drainage or aspiration are the primary therapeutic strategies.

The most common pathogenic agents, which enter the liver by vascular routes, are Escherichia coli, Klebsiella pneumoniae, Salmonella typhi, Proteus vulgaris, streptococci, and staphylococci, but anaerobes may also be present. ${ }^{2}$ Nevertheless, $P$. aeruginosa has long been regarded as a relatively rare pathogen of PLA, especially in patients without underlying conditions, such as lung and renal disease, malignancies, organ transplantation, immunosuppression. ${ }^{4}$ P. aeruginosa was defined as being MDR when the organism was resistant to all agents studied (ampicillin, amoxicillin-clavulanate, ceftriaxone, ceftazidime, cefepime, aztreonam, chloramphenicol, ciprofloxacin, gentamicin, piperacillin, and trimetoprim-sulfamethoxazole), except imipenem, amikacin, and colistina. ${ }^{5}$ When searching Pub Med, we find only one case report of PLA caused by P. aeruginosa, but it was not MDR. ${ }^{4}$ On the other hand, PLA caused by P. aeruginosa was seen $2-6 \%$ in literature, ${ }^{3}$ and this is the first case of PLA caused by community-acquired MDR P. aeruginosa in literature. The incidence of MDR P. aeruginosa increased in recent years. For this reason, we attach great importance to the rational use of antibiotics in inpatients and outpatients.

[Braz J Infect Dis 2010;14(3):218]@Elsevier Editora Ltda.

\section{REFERENCES}

1. Cigarran S, Neches C, Lams JM et al. A case report of pyogenic liver abscess caused by $\mathrm{Fu}$ sobacterium nucleatum in a patient with autosomal dominant polycystic kidney disease undergoing hemodialysis. Ther Apher Dial 2008; 12:91-5.

2. Petri A, Tiszlavicz L, Nagy E et al. Liver abscess caused by Stenotrophomonas maltophilia: Report of a case. Surg Today 2003; 33(3):224-8.
3. Lee KT, Wong SR, Sheen PC. Pyogenic liver abscess: An audit of 10 years' experience and analysis of risk factors. Dig Surg 2001; 18(6):459-65.

4. Lo WT, Wang CC, Hsu ML, Chu ML. Pyogenic liver abscess caused by Pseudomonas aeruginosa in a previously healthy child: report of one case. Acta Paediatr Taiwan 2000; 41(2):98-100.

5. Aloush V, Navan-Venezia S, Seigman-Igra Y, Cabili S, Carmeli Y. Multidrug-resistance Pseudomonas aeruginosa: risk factors and clinical impact. Antimicrob Agents Chemother 2006; 50(1):43-8. 